Mikrochimica Acta [Wien] 1971, 399-404:

(c) by Springer-Verlag 1971

Phoenix Memorial Laboratory, University of Michigan - Ann Arbor, Michigan (U. S. A.), Department of Chemistry, University of Cincinnati Ohio (U. S. A.), Institut de Pharmacie, Université Libre de Bruxelles (Belgium)

\title{
Détermination de traces de mercure dans les systèmes biologiques par activation de neutrons
}

Par

\author{
J. D. Jones, J. M. Rottschafer, H. B. Mark, Jr., K. E. Paulsen, \\ et G. J. Patriarche
}

(Recu le 19 Décembre 19r0)

La détermination de traces de mercure a fait de tous temps l'object de nombreux travaux et la recherche de faibles quantités de cet élément s'est surtout matérialisée dans des domaines très variés tels que: les systèmes biologiques (sang ${ }^{1,2}$, urine ${ }^{4}$, tissus divers ${ }^{3}, 5$, les eaux potables ${ }^{6}$, les médicaments ${ }^{4}$ et même dans l'atmosphère des laboratoires et des usines où cet élément volatil se trouve présent dans l'air en quantités non négligeables ${ }^{1,7}$.

La toxicité, la lenteur d'élimination et la facilité avec laquelle ce toxique se combine avec les groupements $\mathrm{S}-\mathrm{H}$ de certains acides aminés en fait un poison protoplasmique redoutable. Il provoque des lésions particulièrement importantes au niveau rénal et au niveau des glandes salivaires ${ }^{1,8-10}$.

Le problème de dosage du mercure à l'état de traces a été étudié par de nombreux auteurs utilisant les techniques les plus variées et si cette question a été tellement controversée, c'est que les méthodes proposées ne répondent pas exactement aux voeux des chercheurs.

Parmi les nombreux travaux signalons: la méthode classique d'extraction et de titrage à la dithizone ${ }^{2-1,11-13}$ et celle à la $\beta$-naphtylthiocarbazone ${ }^{14}$, celles utilisant des techniques telles que la spectroscopie d'émission ${ }^{15}$, l'absorption atomique ${ }^{16,17,23}$, la fluorescence de rayons $\mathrm{X}^{20}$, la fluorescence d'absorption atomique ${ }^{18,19}$ et les méthodes radiométriques ${ }^{21,22,28}$.

Dans le cas de dosage du mercure dans les liquides et tissus biologiques, il est souvent nécessaire de procéder à une destruction préalable de lá 
matière organique qui allonge de manière considérable la durée de l'opération et fait appel à des réactifs dont la purification a dû être très poussée et à des quantités importantes d'echantillons ${ }^{1,4}$.

Dans ce travail nous proposons une méthode de dosage de traces de mercure se basant sur l'activation de neutrons, suivie d'une séparation réalisée sur résines échangeuses d'anions. Cette technique serait, si nécessaire, suffisamment sensible pour atteindre des quantités à doser de l'ordre d'une partie par billion.

En dépit du coût et des manipulations spéciales inhérentes à la technique, la spécificité et la sensibilité que la méthode par activation de neutrons procure doivent spécialement être mises en valeur. Les résultats obtenus à l'aide de cette technique sont très précis et d'une reproductibilité remarquable.

Il est bien connu et il est important de faire remarquer que les complexes mercuriques sont relativement volatils. Toute destruction ou préconcentration préalable à la méthode analytique choisie entraînent des pertes par volatilisation.

Dans notre cas ces inconvénients sont circonscrits, car l'échantillon est irradié en tubes scellés. Après activation un large excès de mercure qui joue le rôle d'entraîneur est ajouté au contenu du tube et cela préalablement au processus chimique. Durant le traitement chimique, la fraction de mercure radioactif provenant de l'échantillon et qui pourrait être volatilisée est donc complètement négligeable par rapport au reste.

En ce qui concerne la dissolution et la dilution de l'échantillon après irradiation pour traiter le mercuri-chloro complexe $\left(\mathrm{HgCl}_{4}{ }^{{ }^{2}-}\right)$ retenu sur une résine échangeuse, deux méthodes ont été envisagées: celle de Ehman et Huizinga ${ }^{24}$ et celle de Bowen et Gibbons ${ }^{25}$.

Dans ces deux méthodes, les cations interfèrants et plus particulièrement le ${ }^{24} \mathrm{Na}$ ne sont pas adsorbés sur la résine. Dans la méthode de Bowen et $G_{i b b o n s}{ }^{25}$, le complexe $\mathrm{HgCl}_{4}{ }^{2-}$ est élué par l'acide nitrique, précipité sous forme de sulfure de mercure $(\mathrm{HgS})$ et finalement passé au comptage.

Nous avons trouvé qu'il était extrêmement difficile d'obtenir des résultats précis et reproductibles en travaillant de cette manière et que de plus on devait faire appel à de larges volumes d'acide nitrique pour effectuer l'élution.

Nous avons modifié et simplifié ces méthodes en effectuant directement le comptage de la résine réduisant ainsi les manipulations et éliminant diverses sources d'êrreurs. Ce travail rassemble divers résultats obtenus lors de l'analyse du mercure 197 dans des constituants aussi divers que des tissus biologiques, du sang, des cheveux, etc. 
1971/3] Détermination de traces de mercure dans les systèmes biologiques 401

\section{Partie experimentale}

Des prises d'essai de $0,4 \mathrm{~g}$ à $1,0 \mathrm{~g}$ de tissus biologiques $(0,4 \mathrm{à} 1 \mathrm{ml}$ pour les échantillons liquides) sont scellés individuellement dans des tubes en quartz $(10 \mathrm{~cm} \times 0,6 \mathrm{~cm})$. Dans le même temps, un même échantillon est additionné d'une quantité exactement connue de nitrate mercurique qui sert ici de standard interne. Ces deux échantillons sont traités de manière absolument identique et introduits durant 36 heures dans le réacteur sous un flux de neutrons de $10^{13} \mathrm{n} / \mathrm{cm}^{2} / \mathrm{sec}$ (Réacteur Nucléaire Ford du Projet Michigan Memorial Phoenix).

Après irradiation un délai de 2 à 3 jours est respecté en vue d'attendre une décroissance d'activité et de permettre une manipulation plus aisée et plus sûre des échantillons.

Après congélation dans l'azote liquide, chaque ampoule est brisée, le contenu est transféré dans un erlenmeyer et chaque échantillon est additionné de $20 \mathrm{mg}$ de cation mercurique ( $1 \mathrm{ml}$ sol. à $2 \mathrm{~g} \%$ de $\mathrm{Hg}^{2+}$ )

Les fioles coniques sont couvertes à l'aide d'un verre de montre et la destruction de la matière organique restante est effectuée à chaud à l'aide de $5 \mathrm{ml}$ de mélange nitrosulfurique.

Après refroidissement les solutions sont diluées à $25 \mathrm{ml}$ à l'aide d'acide chlorhydrique l-molaire. Ce liquide est passé alors sur une colonne de $10 \mathrm{~cm}$ de résine échangeuse d'ions préalablement traitée par $\mathrm{HCl} 1 \mathrm{M}$ (Dowex 50 à 100 mesh-débit: $5 \mathrm{ml} /$ minute). Le ballon jaugé est rincé à plusieures reprises à l'aide d'acide chlorhydrique $1 M$ et les liquides de rinçage sont passés sur la colonne. De manière à éliminer tout le ${ }^{24} \mathrm{Na}$ présent de la colonne, il est recommandé de faire passer au moins $100 \mathrm{ml}$ d'acide chlorhydrique $1 M$ au travers de la colonne.

La résine est alors retirée de la colonne et placée dans une fiole en verre et comptée durant 400 secondes à l'aide d'un detecteur Camberra (Germanium - Lithium) et d'un analyseur nucléaire à canneaux 4096.

La surface du pic gamma de 77,3 kev du mercure 197 (période: 65 heures) est utilisée pour déterminer la quantité de mercure présent dans l'échantillon et l'étalon interne.

L'activité additionnelle présente dans l'échantillon interne sert à calculer l'activité par microgramme de mercure présent, et cela permet de déterminer la quantité de mercure présente dans l'échantillon.

\section{Résultats et discussion}

Utilisant la technique décrite ci-dessus, nous nous sommes livrés à divers type d'analyses.

En général, les résultats obtenus sur des échantillons contenant entre 0,05 et 50 p. p. m. présentent des déviations standards de $\pm 10 \%$. Les résultats obtenus sont rassemblés au Tableau I. 
Tableau I

Le Tableau I rassemble les résultats représentatifs de dosage de mercure dans divers systèmes

\begin{tabular}{|c|c|c|}
\hline Echantillons & $\mathrm{N}^{0}$ & Résultats (p. p. m.) \\
\hline Poisson Comestible & $\mathrm{I}$ & $0,86 \pm 0,08$ (a) \\
\hline Poisson Comestible & II & $0,58 \pm 0,06(\mathrm{~b}, \mathrm{c})$ \\
\hline Saumon Coho (tissus comestibles) & III & $0,48 \pm 0,05$ \\
\hline Saumon Coho (cerveau) & (III) & 0,23 \\
\hline Saumon Coho (estomac) & (III) & 0,17 \\
\hline Saumon Coho (reins) & (III) & 0,36 \\
\hline Saumon Coho (foies) & (III) & 0,87 \\
\hline Saumon Coho (coeur) & (III) & 0,44 \\
\hline Poisson Blane (tissus, comestibles) & & 0,050 \\
\hline Foie de Faisan & & $<20 \quad$ (p.p. b.) \\
\hline Sang Humain & A & $<5 \quad$ (p.p.b.) \\
\hline Cheveux Humain & $A$ & 1,4 p.p.m. \\
\hline Sang Humain & $\mathrm{B}$ & 19 (p. p. b.) \\
\hline Cheveux Humain & $\mathrm{B}$ & 251 p. p.m. \\
\hline
\end{tabular}

(a) Des dosages effectués sur les mémes échantillons par absorption atomique, fluorescence de rayons $\mathrm{X}$ et d'autres méthodes radiométriques se situaient entre 0,40 et 1,0 p. p.m. ${ }^{20}$ Moyenne 0,74 p. p. m.

(b) La moyenne obtenue sur le méme échantillon par 15 laboratoires différents se situe à 0,51 p. p. m. avec une dispersion comprise entre 0,19 et 1,2 p. p. m. ${ }^{20}$

(c) Une valeur de 0,54 p.p. m. a été obtenue par le National Bureau of Standards qui a utilisé pour la circonstance une méthode radiométrique non destructive ${ }^{27}$.

Il est important de signaler que des expériences effectuées par d'autres méthodes sur les mêmes échantillons ${ }^{20}$ ou sur des échantillons similaires ${ }^{20,26}$, montrent des variations dans les résultats allant d'un facteur 2 à un facteur 5. La méthode que nous proposons ci-dessus est donc beaucoup plus précise.

De plus, il est intéressant de noter que nous pouvons explorer une large gamme de concentration en mercure dans des systèmes les plus variés et ceci sans précautions ni préparatifs particuliers.

De nombreuses expériences nous ont permis de montrer que la limite de dosage la plus basse était de 3 parties par billion. La déviation est alors de $\pm 20 \%$. Si la teneur en mercure se situe aux environs de 1 p. p. $m$. et si l'on dispose de très peu d'échantillon, l'analyse peut s'effectuer à $\pm 20 \%$ sur des échantillons pesant environ $1 \mathrm{mg}$.

Si l'on désire une plus grande précision ou un ultramicrodosage, il est possible de prolonger le temps d'irradiation et le temps de comptage ou encore d'utiliser un flux de neutrons plus puissant. 
1971/3] Détermination de traces de mercure dans les systèmes biologiques 403

\section{Interférences}

L'utilisation d'un résine échangeuse d'ions permet l'élimination des cations présents avec le mercure 197. Si l'élution est suffisante, le ${ }^{24} \mathrm{Na}$ présent en abondance dans les tissus biologiques est parfaitement éliminé.

La seule interférence trouvée est celle $\mathrm{du}^{82} \mathrm{Br}^{-}$qui est également retenu par la résine. Cependant, les concentrations en bromures sont extrêmement minimes dans les milieux que nous avions envisagé d'analyser et leur incidences sur le dosage est faible.

En conclusions, la méthode de dosage de traces de mercure proposée présente de nombreux avantages sur les méthodes décrites antérieurement. L'addition d'entraîneur après irradiation évite les pertes de mercure lors des processus de séparation et lors de la minéralisation. De plus, les modifications et améliorations apportées à la méthode de Bowen et Gibbons raceourcissent considérablement la durée des opérations qui se situe entre 10 et 15 minutes par échantillon.

\section{Remerciements}

Cette recherche a été soutenue en partie par les contrats: F. W. Q. A. $n^{0} 16020$ ELH et N. S. F. $n^{0}$ Ga 25563.

Nous tenons à remercier le Phoenix Memorial Laboratory qui nous a permis de fréquenter ses laboratoires et qui nous a fourni les périodes d'irradiation au réacteur.

\section{Résumé}

Une méthode simple et rapide de détermination de traces de mercure est présentée utilisant l'activation de neutrons. Le mercure 197 sous forme de complexe $\left(\mathrm{HgCl}_{4}{ }^{2-}\right)$ est retenu sur une résine échangeuse d'anion qui est comptée directement. La méthode est appliquée à des milieux biologiques très divers. Les échantillons contiennent des quantités de mercure allant de 10 p. p. b. à 200 p. p. m. La déviation standard dans ces cas est de $\pm 10 \%$.

\section{Summary}

Determination of Traces of Mercury in Biological Material by Neutron Activation

A simple and rapid technique for the trace analysis of mercury by neutron activation is presented. The activated mercury, as an $\mathrm{HgCl}_{4}{ }^{2-}$ complex, is retained on an anion exchange resin which is counted directly. Results obtained for biological samples containing 10 p.p. b. to 200 p.p.m. are given. In this range, results had a $\pm 10 \%$ standard deviation.

\section{Zusammenfassung}

Ein einfaches und rasches Verfahren zur Bestimmung von Spuren Quecksilber durch Neutronenaktivierung wurde beschrieben. Das aktivierte Queck- 
silber wird als $\mathrm{HgCl}_{4}{ }^{2-}$ an einem Anionenaustauscherharz adsorbiert und seine Aktivität direkt gemessen. Biologische Proben mit $10 \mathrm{ppb}$ bis $200 \mathrm{ppm}$ $\mathrm{Hg}$ wurden analysiert. In diesem Bereich betrug die Standardabweichung $\pm 10 \%$.

\section{Bibliographie}

1 C.P. Stewart et A. Stolman, Toxicology, Mechanisms and Analytical Methods, Vol. I, II. New York: Academic Press. 1960.

${ }^{2}$ F. R. Barret, Analyst 81, 294 (1956).

3 P. Rolfe, Analyst 80, 523 (1955).

* F. Lupant-Andre, J. pharm. de Belgique 12, 423, 513 (1967).

5 T. B.Eyl, K. R. Wilcox, Jr., et M. S. Reizen, Mich. Med. 1970, 873.

${ }^{6}$ Chemical and Engineering News 48, No. 43, 11 (1970).

7 G. D. Christian, J. Electroanalyt. Chem. 28, 172 (1969).

8 B. Aberg, L. Ekman, R.Falk, U. Greitz, G. Persson et J. Snihs, Arch. Environ. Health 19, 478 (1969).

${ }^{\circ}$ O. Hook, K. D. Lundgren et A.Swensson, Acta Med. Scand. 150, 131 (1954).

10 H. Matsumoto, G. Koya et T. Takeuchi, J. Neuropathol. Exp. Neurol. 24, 563 (1965).

11 S. Nobel et D. Nobel, Clin. Chem. 4, 150 (1958).

12 T. B. Pierce et P.F. Peck, Analyt. Chim. Acta 26, 557 (1962).

${ }_{13}$ H. Irving et A. M. Kiwan, Analyt. Chim. Acta 45, 243 (1969).

14 R. Meyer, Ann. pharm. Françaises 28, 271 (1970).

15 G. G. Vurek, Analyt. Chemistry 39, 1599 (1967).

${ }_{16}$ D. N. Hingle, G.F. Kirkbright et T. S. West, Analyst 62, 759 (1967).

17 W. R. Hatch et W. C.Ott, Analyt. Chemistry 40, 2082 (1968).

18 M. P. Bratzel, Jr., R. M. Dagnall et J.D. Wineforder, Analyt. Chim. Acta 48, 197 (1969).

19 P. D. Warr, Talanta 17, 543 (1970).

20 R. Grieg, Communication personnelle, Bureau of Commercial Fisheries, Department of Interior U. S. A., 1970.

${ }^{21} H$. D. Livingston, H. Smith et N. Stojanovic, Talanta 14, 505 (1967).

22 Stáry et $R$. Milosévic, Talanta 14, 521 (1967).

${ }^{23}$ C. Thilliez, Chim. analytique 50, 226 (1968).

${ }_{24}$ W.D. Ehman et J.R. Huizinga, Geochim. Cosmochim. Acta 17, 125 (1959).

${ }^{25} H . J$. Bowen et D. Gibbons, Radioactivation Analysis. London: Oxford Press. 1963. p. 264.

${ }^{26}$ D. G. Davis, Communication privée. Louisiana State University - New Orleans U. S. A., 1970.

27 P.D. Lafleur, Communication personnelle, Bureau National des Standards (U. S. A.), 1970.

${ }^{28}$ C.S. Eyer, D. Eyer, D. P. Thibudeaux, E. Klein et J.C. Montalvo, Jr., Communication Southeast-Southwest Combined Regional Meetings - New Orleans, Dec. 2-4, 1970. 